\title{
Living donor liver transplantation for patients with advanced hepatocellular carcinoma
}

\author{
J. Michael Cullen', Paola Vargas ${ }^{1,2}$, Nicolas Goldaracena ${ }^{1,2}$ \\ 'Department of Surgery, University of Virginia, Charlottesville, VA 22908, USA. \\ 2Division of Transplantation, Department of Surgery, University of Virginia, Charlottesville, VA 22908, USA.
}

Correspondence to: Dr. Nicolas Goldaracena, Department of Surgery, University of Virginia, Health System, PO BOX 800709, Charlottesville, VA 22908, USA. E-mail:n.goldaracena@virginia.edu

How to cite this article: Cullen JM, Vargas P, Goldaracena N. Living donor liver transplantation for patients with advanced hepatocellular carcinoma. Hepatoma Res 2020;6:76. http://dx.doi.org/10.20517/2394-5079.2020.69

Received: 23 Jul 2020 First Decision: 24 Aug 2020 Revised: 27 Sep 2020 Accepted: 10 Oct 2020 Published: 6 Nov 2020

Academic Editor: Nobuhisa Akamatsu Copy Editor: Cai-Hong Wang Production Editor: Jing Yu

\begin{abstract}
Liver transplantation (LT) is the treatment of choice for patients with hepatocellular carcinoma (HCC) and underlying liver disease. Given the organ scarcity, LT for patients with HCC have been restricted to those patients associated with the highest survivals. However, many patients with extended criteria HCC can still benefit from LT, but due to deceased organ shortage, they are not offered that opportunity. Living donor liver transplantation (LDLT) emerged as a successful strategy to overcome organ shortage around the world and as LDLT experience grows, this technique might offer the opportunity to expand the indications of LT to patients with advanced HCC. Therefore, since LDLT is not competing for deceased donor organs, many patients with extended criteria HCC who could still benefit from transplantation may have access to this treatment option. In this review, we will discuss the role of LDLT for patients with advanced-stage HCC and how LDLT allows for safe expansion of HCC transplant criteria.
\end{abstract}

Keywords: Living donor liver transplantation, hepatocellular carcinoma, liver transplantation, transplant oncology, clinical outcomes

\section{INTRODUCTION}

Hepatocellular carcinoma (HCC) is the third leading cause of death worldwide and the most common primary liver cancer ${ }^{[1,2]}$. Resection provides the best treatment option for disease confined to the liver.

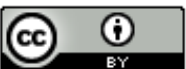

(C) The Author(s) 2020. Open Access This article is licensed under a Creative Commons Attribution 4.0 International License (https://creativecommons.org/licenses/by/4.0/), which permits unrestricted use, sharing, adaptation, distribution and reproduction in any medium or format, for any purpose, even commercially, as long as you give appropriate credit to the original author(s) and the source, provide a link to the Creative Commons license, and indicate if changes were made. 
However, the approach and final treatment depends on tumor size, number of tumors and their location, as well as liver function and performance status. Liver transplantation (LT) has the advantage of removing the underlying liver disease, reducing the risk for postoperative liver failure and de novo HCC development. Therefore, LT is the best treatment option for patients with HCC and underlying liver cirrhosis. However, due to organ scarcity, LT for HCC has been restricted to patients with the highest prospective 5-year survival and recurrence rates ${ }^{[3]}$.

Many have proposed that these criteria might be too restrictive, and that many patients with more advanced disease would still benefit from LT if organs would be available ${ }^{[4,5]}$.

Living donor liver transplantation (LDLT) emerged as a successful strategy to overcome organ shortage around the world ${ }^{[6]}$. As LDLT experience grows, the application of this technique might offer the opportunity to expand the boundaries of LT to settings in which the organ shortage presents a limitation. Because the transplant candidate is not competing for deceased donor organs, patients with extended criteria HCC who could still benefit from LT may have access to the treatment. Moreover, many studies have already proven that LDLT might even offer a benefit over deceased donor liver transplantation (DDLT) to patients fulfilling restrictive transplant criteria ${ }^{[7]}$. It is the scope of this study to review the role of LDLT for patients with more advanced HCC and how LDLT allows for safe expansion of HCC transplant criteria.

\section{Staging systems for hepatocellular carcinoma}

Currently, there is no universal consensus regarding the best staging system for HCC and its management. Among the many proposed, two main classification systems are used. The Barcelona Clinic Liver Cancer (BCLC) classification, the most used in Western countries, and the Hong Kong Liver Cancer (HKLC) staging system, which was created as a treatment guidance for Asian patients with HCC and is mainly used in Asian countries. Both classification systems use parameters such as presence of extrahepatic vascular invasion, tumor size, and number of nodules to stage HCC and provide management recommendations ${ }^{[8-11]}$.

Despite both classifications being widely used, their differences complicate the decision-making process for the transplant team when presented with an advanced HCC. In a recent Brazilian study ${ }^{[9]}, 519$ patients diagnosed with HCC were staged according to the BCLC and HKLC system with the aim to analyze therapeutic approach for different stages. The authors found that between both systems, there was high general agreement regarding therapeutic management of HCC in the Western population. The highest agreement was between stages HKLC-I and BCLC-0 (100\%) and HKLC-IV and BCLC-C (98.7\%). However, agreement was low in intermediate HCC cases (BCLC-B). The authors found that according to the HKLC, more than $50 \%$ of the BCLC-B stage could have been candidates for curative treatment rather than palliative treatment recommended by BCLC. Other authors agreed that BCLC is outdated, highly restrictive with a trend to limit treatment options for more advance tumors, and needs re-evaluation in order to achieve a proper classification of these patients with a management plan according to current practices ${ }^{[10]}$.

It is crucial that HCC staging be conducted in an individualized manner, taking into account biological and etiological heterogeneity among populations in order to provide the patient with the best treatment option available in their case $\mathrm{e}^{[9]}$.

\section{LIVING LIVER DONATION FOR HEPATOCELLULAR CARCINOMA}

Surgical resection is generally recommended for Child-Pugh Class A cirrhotic patients without significant portal hypertension or those with early-stage disease and a single HCC lesion ${ }^{[12]}$. However, multiple studies have shown that transplantation can provide superior long-term outcomes over resection ${ }^{[3,12,13]}$. 
The available literature comparing outcomes of LDLT and DDLT for HCC is limited ${ }^{[14-16]}$. Early reports showed recurrence rates were higher after LDLT than DDLT, but this has been contradicted by recent work $^{[17,18]}$. An important advantage of LDLT is that it effectively allows HCC patients to not be in direct competition with liver failure patients whose Model for End-Stage Liver Disease score afford them higher wait list priority. Goldaracena et al. ${ }^{[16]}$ found patients undergoing LDLT had faster access to transplant and shorter wait times compared with DDLT. In this recent intention-to-treat analysis of 219 LDLTs and 632 DDLTs for HCC, patients with a potential live donor had a 33\% reduction in risk of death from the time of listing due to shorter wait time and decreased waitlist dropout risk. LDLT actually offered a survival benefit over DDLT in this analysis.

Another important aspect unique to LDLT is that each graft is a private gift and not subject to the allocation system. Donation can be direct or altruistic. Patients with advanced HCC should be evaluated on an individual basis so that those outside conventional criteria who would benefit from a transplant can receive one without the risks of disease progression while on the waitlist and its concomitant mortality.

\section{CONVENTIONAL CRITERIA FOR LIVER TRANSPLANT FOR HEPATOCELLULAR CARCINOMA}

The Milan criteria established the benchmark for acceptable outcomes for liver transplantation in HCC. Mazzaferro et al ${ }^{[19]}$ reported recurrence rates less than $15 \%$ and a 5 -year survival of $75 \%$ when transplanting HCC patients with (a) one tumor less than $5 \mathrm{~cm}$ or (b) three tumors each less than $3 \mathrm{~cm}$. The University of California at San Francisco (UCSF) liberalized these size limitations and selected patients with (a) one solitary tumor up to $6.5 \mathrm{~cm}$ or (b) three tumors with the largest $4.5 \mathrm{~cm}$ or less and total tumor diameter $8 \mathrm{~cm}$ or less ${ }^{[20]}$. Using these expanded criteria, the UCSF group matched Milan outcomes with recurrence and 5-year survival rates of $10 \%$ and $75.2 \%$, respectively. In 2008, the Asan Medical Center group in Seoul, South Korea, further expanded eligibility criteria to include patients with up to 5 tumors and size less than $6 \mathrm{~cm}$ and achieved a 5-year survival rate of $81.6 \%{ }^{[21]}$.

\section{TRANSPLANTATION BEYOND CONVENTIONAL CRITERIA}

Many centers have sought to expand these traditional criteria, specifically by emphasizing tumor biology and behavior as opposed to a reliance on tumor size and number ${ }^{[22]}$. The extended Toronto Criteria places no restrictions on number of tumors or tumor size and offers transplantation to patients without systemic cancer-related symptoms, extrahepatic disease, vascular invasion, or poorly differentiated tumors ${ }^{[5]}$. Sapisochin et al. ${ }^{[5]}$ further validated these criteria with a prospective study including 105 patients outside Milan and 76 beyond UCSF criteria, and reported a 5-year survival of $69 \%$ which did not differ significantly from patients within Milan. An important aspect of this study was the authors found an alpha-fetoprotein (AFP) level greater than $500 \mathrm{ng} / \mathrm{mL}$ to be predictive of poor outcomes.

In Asia, LDLT predominates over DDLT, and this region has been an epicenter for criteria expansion. In 2016, the National Cancer Center - Korea reported an $85.2 \%$ overall 5 -year survival and $84 \%$ disease free survival after LDLT for 164 patients with total tumor size of $10 \mathrm{~cm}$ or less and a negative 18 F-fluorodeoxyglucose positron emission tomography scan ${ }^{[23]}$. The Kyushu Criteria, published in 2017, demonstrated a $75.9 \%$ 5-year survival rate when performing LDLT for 161 patients with any number of tumors, but with size less than $5 \mathrm{~cm}$ or des-gamma carboxy prothrombin levels less than $300 \mathrm{mAU} / \mathrm{mL}^{[24]}$. An interesting aspect of this study was that it showed LDLT had a survival benefit when compared with DDLT in an intention to treat sub-analysis, despite LDLT patients having more advanced tumor stage.

\section{LIVING DONOR LIVER TRANSPLANTATION FOR ADVANCED HEPATOCELLULAR CARCINOMA}

Because deceased donor organs are allocated to patients on the waiting list expected to survive the longest after transplant, advanced HCC often precludes DDLT. Many patients outside transplantable criteria are 
treated with palliative therapies that carry a 3 -year survival rate of just $30 \%{ }^{[25]}$. LDLT is now an option for these patients that offers superior outcomes compared with palliative therapy.

A concise pre-operative evaluation is critical. Imaging techniques including CT and MRI are among the most used diagnostic tools. However, characterization of tumor properties such as invasion into segmental branches of the portal vein can be difficult to assess with these modalities alone ${ }^{[26]}$. While biomarkers and cutoff levels vary among centers and within the literature, the most widely used are AFP, PIVKA-II, $\gamma$-GT/ ALT ratio, and recently proposed inflammatory markers including CRP, neutrophil-to-lymphocyte ratio, and platelet-to-lymphocyte ratio ${ }^{[22,26-28]}$.

A multicenter retrospective study in South Korea ${ }^{[28]}$ evaluated the outcomes of LT for extremely advanced HCC patients $(n=169)$, using AFP and PIVKA-II as a selection strategy to decide whether those patients were suitable for LT. The authors found that patients with AFP + PIVKA-II $\leq 300$ showed a 5-year overall and recurrence-free survival rate of $47.8 \%$ and $53.4 \%$, respectively. These values were significantly superior to those of patients with AFP $(\mathrm{ng} / \mathrm{mL})+$ PIVKA-II $(\mathrm{mAU} / \mathrm{mL})>300(21.0 \%$ and $10.8 \%$, respectively; $P<$ 0.001). The authors concluded that that if tumor biology is favorable regardless of tumor size and number, acceptable long-term survival after LT can be expected.

Recently, Kornberg et al. ${ }^{[27]}$ analyzed 119 LT patients with advanced HCC to determine the prognostic impact of clinical and histopathologic factors including pre-LT serum AFP and CRP values. The authors identified that pre-transplant serum levels of AFP $>100 \mathrm{ng} / \mathrm{mL}(\mathrm{OR}=13.31)$ and CRP $>0.8 \mathrm{mg} / \mathrm{dL}(\mathrm{OR}$ $=13.97$ ) were independent predictors of HCC recurrence. The group proposed a serological risk index based on the mentioned biomarkers. A cumulative risk of HCC relapse at 5 years post-LT was determined to be $2.3 \%$ in low serological tumor activity (STA) index (AFP $\leq 100 \mathrm{ng} / \mathrm{mL}+\mathrm{CRP} \leq 0.8 \mathrm{mg} / \mathrm{dL}$ ), $17.1 \%$ in intermediate STA (AFP $\leq 100 \mathrm{ng} / \mathrm{mL}$ or CRP $\leq 0.8 \mathrm{mg} / \mathrm{dL}$ ), and $91.6 \%$ in high STA index (AFP $>100 \mathrm{ng} / \mathrm{mL}$ $+\mathrm{CRP}>0.8 \mathrm{mg} / \mathrm{dL} ; P<0.001)$. Prospective randomized studies that provide validation and present reproducible results are needed.

Despite being a promising therapeutic approach for advance HCC, evidence suggest that absolute contraindications for transplant, includes presence of extrahepatic metastasis such as suspicious porta hepatic nodal diseases, massive infiltrative type, major vascular invasion, and cases with ruptured HCC ${ }^{[29,30]}$.

\section{Vascular invasion}

Portal vein tumor thrombosis (PVTT) is seen in up to $60 \%$ of HCC patients, and considered a marker of systemic disease associated with a high rate of post-transplant recurrence ${ }^{[31-33]}$. Because of this, PVTT has been traditionally considered a contraindication to liver transplantation. However, new studies are challenging this convention based upon the PVTT classification. There are several classifications for PVTT based on the portal vein segment involved. However, two classifications are currently valid to effectively assess prognosis and provide guidance for surgical treatment ${ }^{[34]}$. The Liver Cancer Study Group of Japan differentiates PVTT in four main categories of portal vein invasion, VP1, VP2, VP3, and VP4 ${ }^{[34-36]}$. In a similar fashion, Cheng et al ${ }^{[34]}$ proposed a four-category classification, with the distinction of a category for microscopic portal vein invasion and the allusion of the extension of PVTT involving the superior mesenteric vein or inferior vena cava ${ }^{[37,38]}$ [Figure 1].

The prognosis of patients with HCC and PVTT is known to be deleterious ${ }^{[39]}$, and the BCLC recommendation indicates Sorafenib as the only treatment option ${ }^{[33,40]}$. However, depending on the PVTT classification, different treatment options are available and survival rates vary among the literature. A summary of treatment strategies according to PVTT is provided in Figure $1^{[26,34,35,38,41]}$. In terms of LDLT, within the scope of the present review, recent studies demonstrated LDLT is a viable option to improve 


\section{Most commonly used classifications for Portal Vein Tumor Thrombosis}

Cheng's classification of portal vein tumor thrombosis (37)

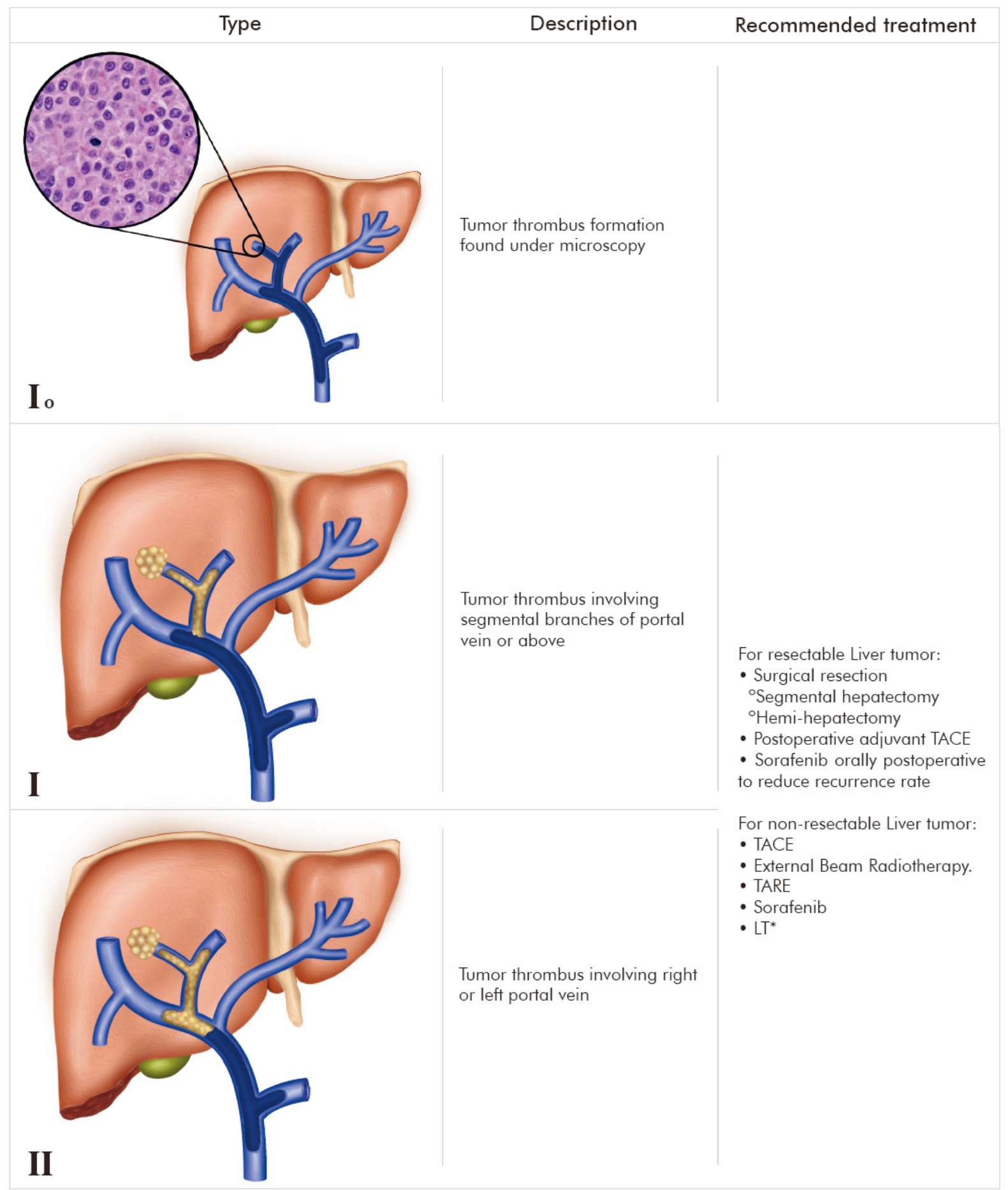




\begin{tabular}{|c|c|c|}
\hline Type & Description & Recommended treatment \\
\hline II & $\begin{array}{l}\text { Tumor thrombus involving the } \\
\text { main portal vein trunk }\end{array}$ & $\begin{array}{l}\text { For resectable Liver tumor: } \\
\text { - Operative treatment only after } \\
\text { downstaging via preoperative } \\
\text { radiotherapy. } \\
\text { oHemi-hepatectomy } \\
\text { oHepatectomy plus thrombectomy, } \\
\text { embolectomy or en bloc resection } \\
\text { (with or without reconstruction of the } \\
\text { main portal vein) } \\
\text { For non-resectable Liver tumor: } \\
\text { - TACE (under specific conditions, } \\
\text { controversial use). } \\
\text { - External Beam Radiotherapy } \\
\text { - TARE } \\
\text { - Sorafenib } \\
\text { - LT* }\end{array}$ \\
\hline 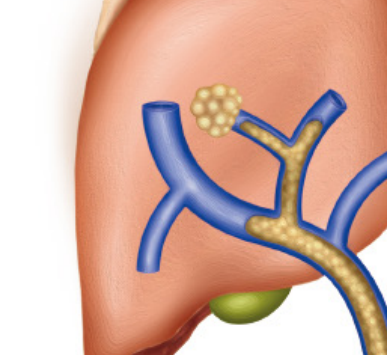 & $\begin{array}{l}\text { Tumor thrombus involving the } \\
\text { superior mesenteric vein }\end{array}$ & $\begin{array}{l}\text { - External Beam radiotherapy. } \\
\text { - TARE } \\
\text { - Sorafenib } \\
\text { **Not enough support: } \\
\text { - Systemic chemotherapy using } \\
\text { different regimens including: } \\
\text { 5-FU, cisplatin, leucovorin, } \\
\text { methotrexate, IFN-alpha 2b. } \\
\text { - LT* }\end{array}$ \\
\hline $\mathbf{I V}$ & & \\
\hline
\end{tabular}

Japan's PVTT portal vein invasion (VP) classification $(34,35)$

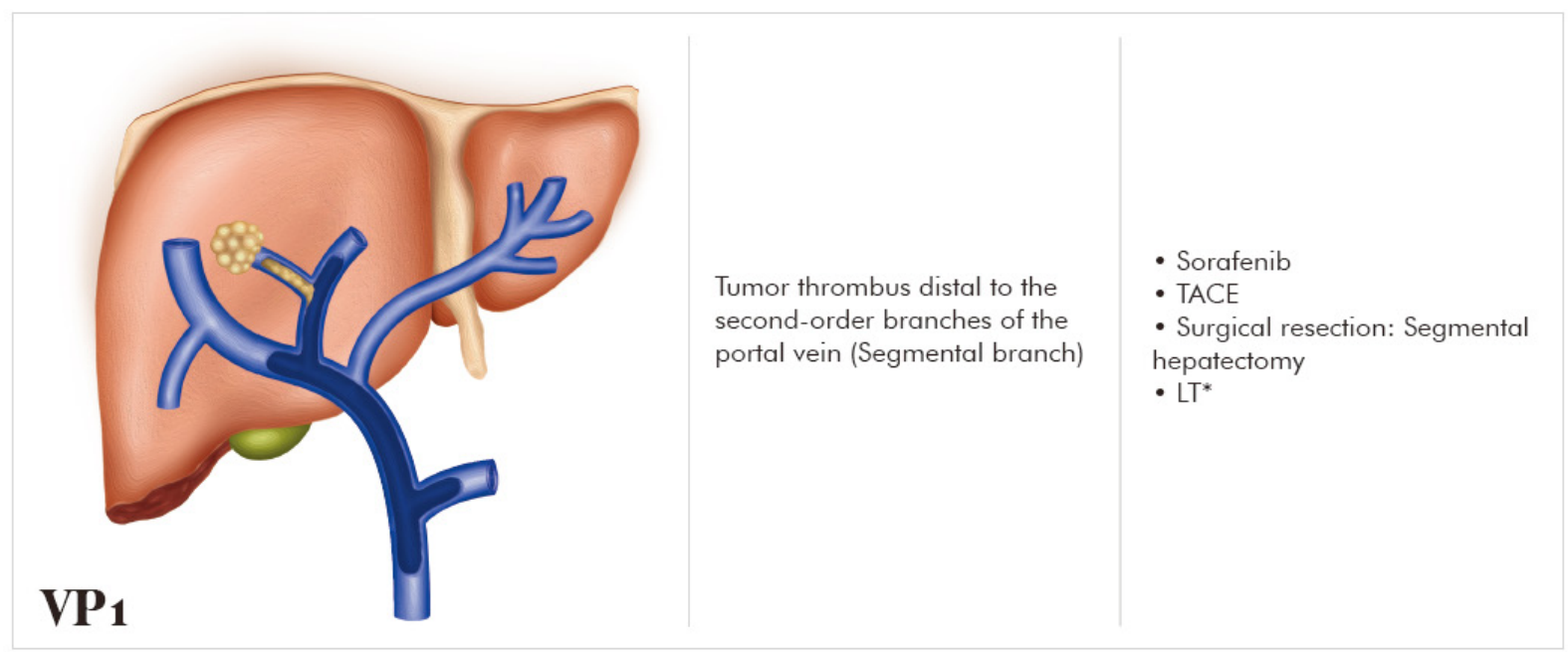




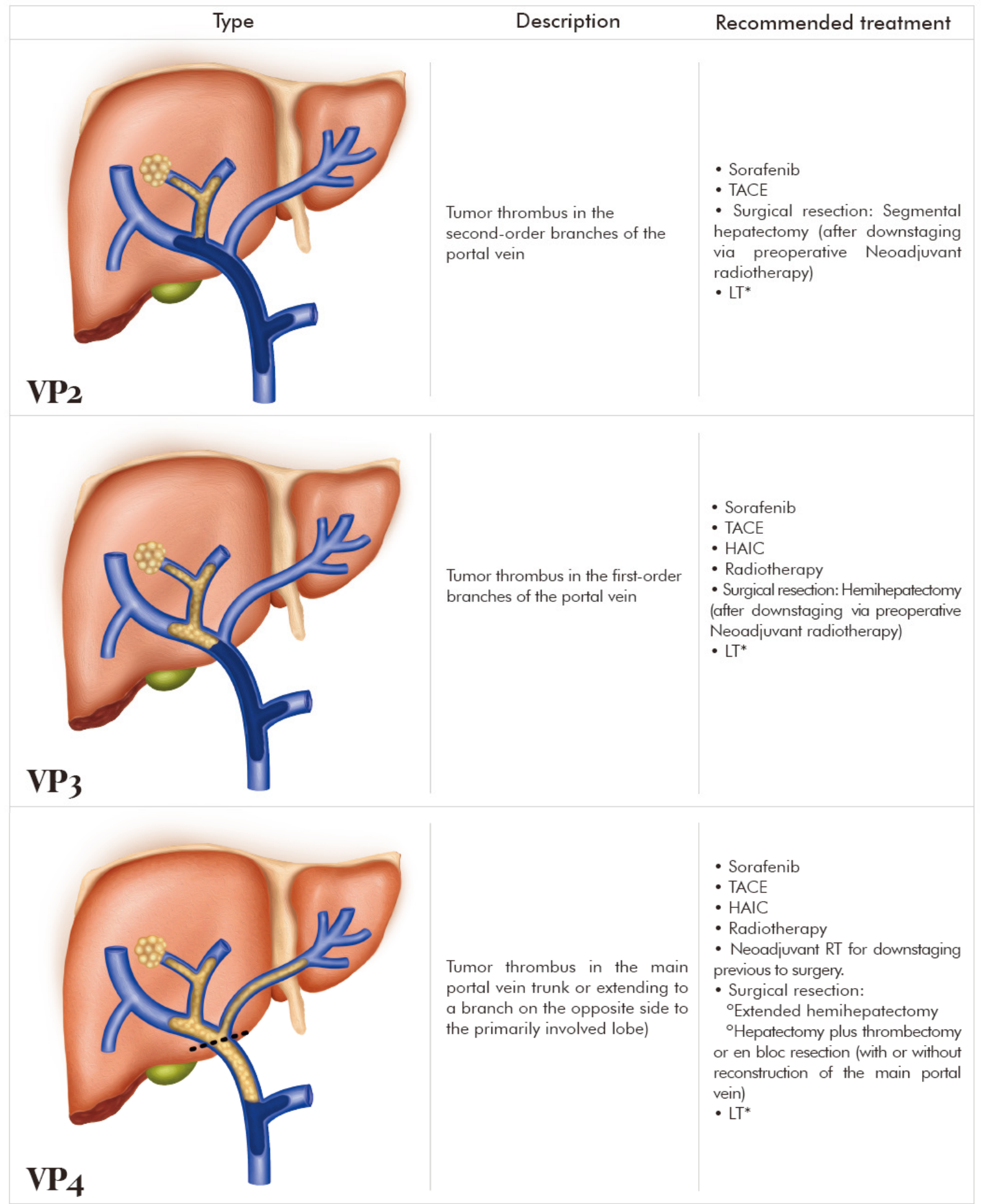

Figure 1. Most commonly used classifications for Portal Vein Tumor Thrombosis (PVTT). Cheng's classification of portal vein tumor thrombosis ${ }^{[37]}$, Japan's PVTT portal vein invasion (VP) classification ${ }^{[34,35]}$. HAIC: hepatic arterial infusion chemotherapy; IFN-alpha $2 \mathrm{~b}$ : interferon alfa-2b; LT: liver transplantation; RT: radiation therapy; TACE: transarterial chemoembolization; TARE: transarterial radioembolization; 5-FU: 5-fluorouracil. *Under certain conditions; ${ }^{* \star}$ not enough support ${ }^{[26,34,35,38,37,40,41]}$ 
long-term survival outcomes among carefully selected patients under specific criteria such as: (a) PVTT in a segmental branch, (b) AFP level $<100 \mathrm{ng} / \mathrm{mL}$, (c) low AP score, and (d) patients with HCC and PVTT who were successfully downstaged ${ }^{[36,42,43]}$.

In a paper in 2017, Choi et al. ${ }^{[36]}$ evaluated PVTT recurrence, disease-free survival (DFS), and overall survival (OS) in a cohort undergoing LDLT to treat HCC. Among the 242 patients, microvascular invasion was apparent in two patients, segmental PVTT was noted in 27, while lobar PVTT was seen in 7 patients. The authors aimed to define the branch level in which PVTT presence was acceptable in LT and found no difference in DFS rate between microvascular invasion and segmental PVTT group, while those with lobar PVTT exhibited poorer DFS and OS. No significant difference in the maximal or total tumor diameter or tumor number was detected between groups. The authors found that in the segmental PVTT group, the 5-year DFS and OS rates were $63.9 \%$ and 50.3\%, respectively, and did not differ significantly from those of the microvascular invasion group. Based on these results and similar 5-year survival rates between patients, transplantation should not be restricted, and mutual donor and recipient interest should guide decisions to proceed with transplantation.

Other LDLT experiences with patients with vascular involvement include the one by Lee et al ${ }^{[43]}$, which reported successful LDLT in 11 HCC patients with macrovascular invasion. Recurrence-free survival at 5 years was $45.5 \%$, and 5-year overall survival was $63.6 \%$. The authors found tumor thrombus extension into the main portal vein, elevated AFP, and tumor size greater than $7 \mathrm{~cm}$ to be independent risk factors for recurrence. In the largest study to date, 46 patients with PVTT underwent LDLT after a downstaging protocol using stereotactic body radiation in combination with transarterial chemo-embolization or transarterial radio-embolization ${ }^{[42]}$. Five-year recurrence free survival and overall survival rates were $51 \%$ and $57 \%$, respectively. Furthermore, the authors found several favorable prognosticators, including lower initial AFP levels, significant decrease in AFP after downstaging, and low tumor grade. The authors proposed offering their downstaging protocol to "all comers" with HCC and PVTT without extrahepatic disease or extensive thrombosis of the main portal or superior mesenteric veins. Once again, this offers the surgeon an opportunity to maximize the benefit of LDLT by allowing timing of the operation when the disease is most under control and when it is most likely to be successful for the recipient.

\section{Strategies before and after LDLT in advanced HCC patients}

Downstaging and adjuvant therapy administration are commonly used strategies in advanced HCC patients ${ }^{[44,45]}$. The goals of each vary depending on the treatment modality to be performed ${ }^{[44]}$. When applied before transplantation (versus before resection), downstaging strategies are used to convert a patient outside accepted criteria for transplantation into an acceptable candidate, by reducing tumor burden. It is also used as a way to select patients with low rates of recurrence among those who would have been excluded based on current criteria. On the other hand, adjuvant therapy is used in patients following surgical management to help decrease the incidence of HCC recurrence, and thus, improve their outcomes $^{[30,44,4]}$.

\section{Locoregional therapies}

Locoregional therapies provide several benefits: tumors outside Milan can be downstaged or downsized so that criteria are met, patients within Milan with disease progression can be bridged to avoid waitlist dropout, and tumor necrosis can be induced which reduces intraoperative tumor spread. Additionally, response to these therapies reflects favorable disease biology and indicates recurrence risk.

The most commonly used locoregional techniques are stereotactic body radiation, transarterial chemoembolization, transarterial radio-embolization, and radiofrequency ablation. Aravinthan et al. ${ }^{[46]}$ reported their outcomes after liver transplantation in patients with advanced HCC within extended Toronto Criteria. 
Sixty-five percent of the patients underwent a protocol of bridging therapy, which led to significant reductions in tumor burden and disease progression prior to transplant. A recurrence rate of $35 \%$ was observed and 3-year survival was 56\%, representing superior survival compared with palliative treatments. Positive outcomes were also reported by Jeong et al ${ }^{[47]}$ in their experience with 17 HCC patients with major vascular invasion who underwent LDLT after combined treatment with transarterial chemo-embolization and radiotherapy. The authors found that the 1- and 3-years DFS were $70.6 \%$ and $57.8 \%$ and the OS rates at 1 - and 3-years were $87.4 \%$ and $60.5 \%$, respectively. Even though long-term results are not provided, the authors concluded that acceptable oncologic outcomes can be achieved in select HCC patients with major vascular invasion, and suggest that LDLT might be a therapeutic option for these patients if the tumors are downstaged considering that 1-year survival rate for HCC patients with PVTT is less than $10 \%$. Even though it has been reported that the use of downstaging strategies in the presence of extended criteria of HCC for LDLT has decreased in Asian countries, published experience among Asian institutions revealed comparable 5-year overall survival rates in patients who received LDLT after downstaging versus those without downstaging ${ }^{[48]}$.

A benefit to pre-operative therapy is the additional time for proper surgical planning. Where the price of DDLT might be a gap in planning, locoregional therapies create an ideal setting for LDLT in that it provides better selection and timing, allowing for a set date for the surgery when it is most likely to succeed when the disease response is most appropriate after locoregional therapy.

\section{Adjuvant therapy}

HCC recurrence remains the leading cause of death after liver transplantation, and patients with advanced disease are at particular risk ${ }^{[49]}$. Early recurrences are commonly seen in patients with associated negative prognosis factors such as vascular invasion ${ }^{[4]}$. In order to help decrease the incidence of HCC recurrence post-transplantation, numerous strategies have been proposed as adjuvant therapy; however, none have been able to provide enough support regarding prolonged recurrence-free survival and none have been widely accepted ${ }^{[50]}$. Other factors complicating the standardization of an adjuvant regimen for HCC is the heterogeneity among HCC between different regions. Thus, targeting recurrence risk factors should be analyzed in an individual manner. Currently published evidence include experience with systemic therapy such as oral anti-HBV agents, tyrosine kinase inhibitors, IFN-alpha, TACE, anti-PD-1 antibodies (e.g., Nivolumab, pembrolizumab, atezolizumab), molecular-targeted agents, and chemotherapy agents (e.g., Oxaliplatin plus fluorouracil/leucovorin $)^{[50]}$.

Select immunosuppressive medications may portend an oncologic benefit, specifically mammalian target of rapamycin (mTOR) inhibitors such as everolimus and sirolimus ${ }^{[51,52]}$. Inhibition of tumor growth mediated by properties such as antiangiogenic and antiproliferative effects makes mTOR an extraordinary class of immunosuppressants ${ }^{[53]}$. In 2016, a prospective-randomized open-label international trial compared recurrence-free survival in sirolimus-containing versus mTOR inhibitor-free immunosuppression patients undergoing LT for HCC. The authors found that sirolimus in LT recipients with HCC did not improve long-term recurrence free survival beyond 5 years. However, a benefit in the first 3 to 5 years for recurrence free survival and OS was evident. Regarding adverse events, both groups reported similar frequencies ${ }^{[5,54]}$.

Clinicians should be aware of the increased risk of post-transplant HCC recurrence with calcineurin inhibitors ${ }^{[54]}$. In a systematic review of 42 publications, patients on everolimus had significantly lower recurrence rates of HCC versus calcineurin inhibitors and sirolimus ${ }^{[52]}$. Sorafenib, a multikinase inhibitor, has also shown promise as an adjuvant therapy. In a study of 30 patients with HCC beyond Milan, sorafenib reduced recurrence of post-transplant HCC compared with capecitabine ${ }^{[55]}$. However, contrasting experiences have been published. A recent publication evaluating the role of Sorafenib on HCC recurrence and survival in 45 patients with advance HCC on explant pathology after LT found that adjuvant 
treatment with sorafenib in LT recipients with high-risk features does not improved HCC recurrence-free or overall survival ${ }^{[56]}$. When used, it is crucial to be aware of reported early and severe toxicities such as hepatotoxicity, thrombocytopenia, anorexia, fatigue, hand-foot syndrome, and diarrhea ${ }^{[57]}$.

An ongoing phase 3 study [IMbrave150 study (NCT03434379)] is being done to compare atezolizumab with bevacizumab combination therapy with the standard of care (Sorafenib) among a large cohort of patients with systemic treatment-naïve unresectable HCC. Preliminary phase Ib results showed that the proposed combination resulted in improved progression-free survival compared with atezolizumab monotherapy and had a tolerable safety profile ${ }^{[58]}$. Other clinical trials evaluating treatment combinations with acceptable safety profile are currently being developed ${ }^{[5]}$.

While promising, additional studies will be necessary to further elucidate the potential benefits of specific immunosuppressive regimens as an adjuvant therapy after LDLT for advanced HCC.

\section{Recurrence risk surveillance and useful prognosis tools}

Tumor recurrence after LT is seen in approximately $15 \%-20 \%$ of HCC patients despite being within the Milan criteria ${ }^{[54]}$. These data are even more worrisome in patients with advanced HCC, which supports the notion that identification of risk factors for recurrence and a proper evaluation for LT plays a pivotal role in the management of these patients.

Risk factors related to recurrence of HCC after LT include tumor factors such as staging, vascular invasion, differentiation, and AFP level, among others. Relevant patient factors such as obesity, viral etiology, HCV treatment, and NAFLD are also important, as well as factors related to the transplant, including percutaneous tumor biopsy, waiting time, bridging therapy, donor's age, ischemia time, surgical technique, and post-transplant immunosuppression regimen and adjuvant therapy.

There is no consensus regarding the protocol for monitoring recurrence after LT. However, important scores have been proposed and should be taken into account when performing follow-up for these patients $^{[60]}$.

\section{MORAL score}

The Model of Recurrence After Liver (MORAL) transplant for HCC is a score developed by Halazun et al. ${ }^{[61]}$ at Columbia University Medical Center to predict recurrence and risk-stratification pre- and postoperatively. Variables included in the MORAL scoring system include pre-operative NLR $\geq 5$, maximum AFP $>200 \mathrm{ng} / \mathrm{mL}$, largest tumor size $>3 \mathrm{~cm}$, grade 4 tumors, vascular invasion, largest size on path $>3 \mathrm{~cm}$, and tumor number on path $>3$. The recurrence risk stratification according to Pre-MORAL score divide the risk of recurrence in low risk (0-2), medium risk (3-6), high risk (7-10) and very high risk (>10).

Other authors, including a group from South Korea, have published variations of this model. Their variation was derived using PIVKA II levels and AFP to stratify risk and predict HCC recurrence and was developed including 205 patients with HCC beyond Milan criteria undergoing LDLT. The authors found that a low MORAL score $(\leq 314.8)$ was associated with significantly longer recurrence-free and overall survivals in the beyond Milan criteria group. The 5-year recurrence-free and OS rates among these patients were $66.3 \%$ and $82.6 \%$, respectively. They conclude that their model provides advanced prognostication and support the idea that HCC patients beyond Milan criteria without extrahepatic metastasis and low MORAL score might be potential candidates for LDLT ${ }^{[62]}$.

\section{Metroticket 2.0 model}

The model for Analysis of Competing Risks of Death After Liver Transplantation (Metroticket 2.0) for HCC is a web-based calculator recognized by the European Liver and Intestine Transplant Association 
and the International Liver Transplantation, developed by Mazzaferro et al ${ }^{[63]}$. The tool is used to predict post-transplantation HCC specific survival, considering as events of interest only those deaths caused by tumor recurrence. The objective is to provide an upgraded prognostic tool and refine selection criteria for liver transplantation in patients with HCC. Outcome prediction was conducted using pre-operative determinants of prognosis frequently associated with higher risk of pre-transplantation tumor progression and poorer post-transplantation survival. The calculator is a free accessible tool found at www.hcc-oltmetroticket.org ${ }^{[63]}$.

\section{CONCLUSION}

Until proven otherwise, surgical resection is the treatment of choice for patients with HCC confined to the liver. It has been shown that even patients with advanced HCC can achieve cure with a more radical resection as liver transplantation offers. Therefore, it is crucial that the transplant community changes the paradigm and improves transplant criteria for patients with HCC. We should focus more on tumor biology than size restrictions. In addition, tumor markers such as AFP and pathologic grade must be assessed. Response to pre-transplant locoregional therapies might be a surrogate marker of more favorable disease, which may impart lower post-transplant recurrence risk. In addition, current data proposes that PVTT should not necessarily be considered a contraindication.

However, the question as to who should receive a liver transplant still persists. An important component to answering this question will be the transplant surgeons who continue to push the limits and boundaries by offering liver transplantation to advanced HCC patients. Nevertheless, the most crucial components to what can be achieved are the patients and the donors; their willingness to accept the risks will make it possible.

\section{DECLARATIONS}

\section{Acknowledgments}

The authors acknowledge Andres Ramos for providing assistance with the illustrations in this manuscript.

\section{Authors' contributions}

Participated in conception and design of the study; generation, collection, assembly, interpretation and critical revision of the data; drafting of the manuscript; approval of the final version of the manuscript: Cullen JM

Participated in the generation, collection, assembly, interpretation and critical revision of the data; drafting of the manuscript; approval of the final version of the manuscript: Vargas $\mathrm{P}$

Participated in conception and design of the study; generation, collection, assembly, interpretation and critical revision of the data; drafting of the manuscript; critical revision of the manuscript for important intellectual content; approval of the final version of the manuscript: Goldaracena N

\section{Availability of data and materials}

Not applicable.

\section{Financial support and sponsorship}

None.

\section{Conflicts of interest}

All authors declared that there are no conflicts of interest.

\section{Ethical approval and consent to participate}

Not applicable. 


\section{Consent for publication}

Not applicable.

\section{Copyright}

(c) The Author(s) 2020.

\section{REFERENCES}

1. Ferlay J, Soerjomataram I, Dikshit R, et al. Cancer incidence and mortality worldwide: sources, methods and major patterns in GLOBOCAN 2012. Int J Cancer 2015;136:E359-86.

2. Berumen J, Hemming A. Liver transplantation for hepatocellular carcinoma. Abdom Radiol (NY) 2018;43:185-92.

3. Proneth A, Zeman F, Schlitt HJ, Schnitzbauer AA. Is resection or transplantation the ideal treatment in patients with hepatocellular carcinoma in cirrhosis if both are possible? A systematic review and metaanalysis. Ann Surg Oncol 2014;21:3096-107.

4. Mulligan DC. The ongoing quest to find the appropriate patients to transplant with hepatocellular carcinoma: Milan to san Francisco to Toronto and beyond. Hepatology 2016;64:1853-5.

5. Sapisochin G, Goldaracena N, Laurence JM, et al. The extended Toronto criteria for liver transplantation in patients with hepatocellular carcinoma: a prospective validation study. Hepatology 2016;64:2077-88.

6. Raia S, Nery JR, Mies S. Liver transplantation from live donors. Lancet 1989;2:497.

7. Wong TCL, Ng KKC, Fung JYY, et al. Long-term survival outcome between living donor and deceased donor liver transplant for hepatocellular carcinoma: intention-to-treat and propensity score matching analyses. Ann Surg Oncol 2019;26:1454-62.

8. Cheung TTT, Kwok PCH, Chan S, et al. Hong Kong consensus statements for the management of unresectable hepatocellular carcinoma. Liver Cancer 2018;7:40-54.

9. de Freitas LBR, Longo L, Santos D, Grivicich I, Álvares-da-Silva MR. Hepatocellular carcinoma staging systems: Hong Kong liver cancer vs Barcelona clinic liver cancer in a Western population. World J Hepatol 2019;11:678-88.

10. De Souza Martins Fernandes E, Rodrigues PD, Álvares-da-Silva MR, et al. Treatment strategies for locally advanced hepatocellular carcinoma. Transl Gastroenterol Hepatol 2019;4:12.

11. Xie DY, Ren ZG, Zhou J, Fan J, Gao Q. 2019 Chinese clinical guidelines for the management of hepatocellular carcinoma: updates and insights. Hepatobiliary Surg Nutr 2020;9:452-63.

12. Iwatsuki S, Starzl TE, Sheahan DG, et al. Hepatic resection versus transplantation for hepatocellular carcinoma. Ann Surg 1991;214:2219 .

13. Bigourdan JM, Jaeck D, Meyer N, et al. Small hepatocellular carcinoma in Child A cirrhotic patients: hepatic resection versus transplantation. Liver Transpl 2003;9:513-20.

14. Bhangui P, Vibert E, Majno P, et al. Intention-to-treat analysis of liver transplantation for hepatocellular carcinoma: living versus deceased donor transplantation. Hepatology 2011;53:1570-9.

15. Azoulay D, Audureau E, Bhangui P, et al. Living or brain-dead donor liver transplantation for hepatocellular carcinoma. Ann Surg 2017;266:1035-44.

16. Goldaracena N, Gorgen A, Doyle A, et al. Live donor liver transplantation for patients with hepatocellular carcinoma offers increased survival vs. deceased donation. J Hepatol 2019;70:666-73.

17. Xiao GQ, Song JL, Shen S, Yang JY, Yan LN. Living donor liver transplantation does not increase tumor recurrence of hepatocellular carcinoma compared to deceased donor transplantation. World J Gastroenterol 2014;20:10953-9.

18. Fisher RA, Kulik LM, Freise CE, et al. Hepatocellular carcinoma recurrence and death following living and deceased donor liver transplantation. Am J Transplant 2007;7:1601-8.

19. Mazzaferro V, Regalia E, Doci R, et al. Liver transplantation for the treatment of small hepatocellular carcinomas in patients with cirrhosis. N Engl J Med 1996;334:693-9.

20. Yao FY, Ferrell L, Bass NM, et al. Liver transplantation for hepatocellular carcinoma: expansion of the tumor size limits does not adversely impact survival. Hepatology 2001;33:1394-403.

21. Lee SG, Hwang S, Moon DB, et al. Expanded indication criteria of living donor liver transplantation for hepatocellular carcinoma at one large-volume center. Liver Transpl 2008;14:935-45.

22. Cillo U, Giuliani T, Polacco M, et al. Prediction of hepatocellular carcinoma biological behavior in patient selection for liver transplantation. World J Gastroenterol 2016;22:232-52.

23. Lee SD, Lee B, Kim SH, et al. Proposal of new expanded selection criteria using total tumor size and (18)F-fluorodeoxyglucose - positron emission tomography/computed tomography for living donor liver transplantation in patients with hepatocellular carcinoma: The National Cancer Center Korea criteria. World J Transplant 2016;6:411-22.

24. Uchiyama H, Itoh S, Yoshizumi T, et al. Living donor liver transplantation for hepatocellular carcinoma: results of prospective patient selection by Kyushu University Criteria in 7 years. HPB (Oxford) 2017;19:1082-90.

25. Llovet JM, Real MI, Montaña X, et al. Arterial embolisation or chemoembolisation versus symptomatic treatment in patients with unresectable hepatocellular carcinoma: a randomised controlled trial. Lancet 2002;359:1734-9.

26. Wang JC, Xia AL, Xu Y, Lu XJ. Comprehensive treatments for hepatocellular carcinoma with portal vein tumor thrombosis. $J$ Cell Physiol 2019;234:1062-70. 
27. Kornberg A, Schernhammer M, Kornberg J, Friess H, Thrum K. Serological risk index based on alpha-fetoprotein and C-reactive protein to indicate futile liver transplantation among patients with advanced hepatocellular carcinoma. Dig Dis Sci 2019;64:269-80.

28. Lee HW, Song GW, Lee SG, et al. Patient selection by tumor markers in liver transplantation for advanced hepatocellular carcinoma. Liver Transpl 2018;24:1243-51.

29. Lin CC, Chen CL. Living donor liver transplantation for hepatocellular carcinoma achieves better outcomes. HepatoBiliary Surg Nutr 2016;5:415-21.

30. Lin C, Elsarawy AMAA, Chen C. Living donor liver transplantation for hepatocellular carcinoma. In: Abdeldayem HM, editor. InTech; 2017.

31. Minagawa M, Makuuchi M. Treatment of hepatocellular carcinoma accompanied by portal vein tumor thrombus. World J Gastroenterol 2006;12:7561-7.

32. Lee YH, Hsu CY, Huang YH, et al. Vascular invasion in hepatocellular carcinoma: prevalence, determinants and prognostic impact. $J$ Clin Gastroenterol 2014;48:734-41.

33. Chen ZH, Zhang XP, Lu YG, et al. Actual long-term survival in HCC patients with portal vein tumor thrombus after liver resection: a nationwide study. Hepatol Int; 2020.

34. Cheng S, Yang J, Shen F, et al. Multidisciplinary management of hepatocellular carcinoma with portal vein tumor thrombus - Eastern Hepatobiliary Surgical Hospital consensus statement. Oncotarget 2016;7:40816-29.

35. Cerrito L, Annicchiarico BE, Iezzi R, et al. Treatment of hepatocellular carcinoma in patients with portal vein tumor thrombosis: Beyond the known frontiers. World J Gastroenterol 2019;25:4360-82.

36. Choi HJ, Kim DG, Na GH, et al. The clinical outcomes of patients with portal vein tumor thrombi after living donor liver transplantation. Liver Transpl 2017;23:1023-31.

37. Shi J, Lai EC, Li N, et al. A new classification for hepatocellular carcinoma with portal vein tumor thrombus. $J$ Hepatobiliary Pancreat Sci 2011;18:74-80

38. Peng SY, Wang XA, Huang CY, et al. Better surgical treatment method for hepatocellular carcinoma with portal vein tumor thrombus. World J Gastroenterol 2018;24:4527-35.

39. Huo L, Wei W, Yan Z, et al. Short-term and long-term outcomes of liver resection for HCC patients with portal vein tumor thrombus. Cell Biosci 2019;9:23.

40. Cheng S, Chen M, Cai J; National Research Cooperative Group for Diagnosis and Treatment of Hepatocellular Carcinoma with Tumor Thrombus. Chinese expert consensus on multidisciplinary diagnosis and treatment of hepatocellular carcinoma with portal vein tumor thrombus: 2016 edition. Oncotarget 2017;8:8867-76.

41. Moriguchi M, Furuta M, Itoh Y. A review of non-operative treatments for hepatocellular carcinoma with advanced portal vein tumor thrombus. J Clin Transl Hepatol 2017;5:177-83.

42. Soin AS, Bhangui P, Kataria T, et al. Experience with LDLT in patients with hepatocellular carcinoma and portal vein tumor thrombosis postdownstaging. Transplantation 2020; doi: 10.1097/TP.0000000000003162.

43. Lee KW, Suh SW, Choi YR, et al. Macrovascular invasion is not an absolute contraindication for living donor liver transplantation. Liver Transplant 2017;23:19-27.

44. Toso C, Mentha G, Kneteman NM, Majno P. The place of downstaging for hepatocellular carcinoma. $J$ Hepatol 2010;52:930-6.

45. Akateh C, Black SM, Conteh L, et al. Neoadjuvant and adjuvant treatment strategies for hepatocellular carcinoma. World J Gastroenterol 2019;25:3704-21.

46. Aravinthan AD, Bruni SG, Doyle AC, et al. Liver transplantation is a preferable alternative to palliative therapy for selected patients with advanced hepatocellular carcinoma. Ann Surg Oncol 2017;24:1843-51.

47. Jeong Y, Shin MH, Yoon SM, et al. Liver transplantation after transarterial chemoembolization and radiotherapy for hepatocellular carcinoma with vascular invasion. $J$ Gastrointest Surg 2017;21:275-83.

48. Yoon YI, Lee SG. Living donor liver transplantation for hepatocellular carcinoma: an asian perspective. Dig Dis Sci 2019;64:993-1000.

49. Todo S, Furukawa H; Japanese Study Group on Organ Transplantation. Living donor liver transplantation for adult patients with hepatocellular carcinoma: experience in Japan. Ann Surg 2004;240:451-9.

50. Zhu XD, Li KS, Sun HC. Adjuvant therapies after curative treatments for hepatocellular carcinoma: current status and prospects. Genes Dis 2020;7:359-69.

51. Toso C, Meeberg GA, Bigam DL, et al. De novo sirolimus-based immunosuppression after liver transplantation for hepatocellular carcinoma: long-term outcomes and side effects. Transplantation 2007;83:1162-8.

52. Cholongitas E, Mamou C, Rodríguez-Castro KI, Burra P. Mammalian target of rapamycin inhibitors are associated with lower rates of hepatocellular carcinoma recurrence after liver transplantation: a systematic review. Transpl Int 2014;27:1039-49.

53. Geissler EK, Schnitzbauer AA, Zölke C, et al. Sirolimus use in liver transplant recipients with Hepatocellular carcinoma: a randomized, Multicenter, open-label phase 3 Trial. Transplantation 2016;100:116-25.

54. Filgueira NA. Hepatocellular carcinoma recurrence after liver transplantation: Risk factors, screening and clinical presentation. World $J$ Hepatol 2019;11:261-72.

55. Huang L, Li GM, Zhu JY, et al. Efficacy of sorafenib after liver transplantation in patients with primary hepatic carcinoma exceeding the Milan criteria: a preliminary study. Onco Targets Ther 2012;5:457-62.

56. Satapathy SK, Das K, Kocak M, et al. No apparent benefit of preemptive sorafenib therapy in liver transplant recipients with advanced hepatocellular carcinoma on explant. Clin Transplant 2018;32:e13246.

57. Noda S, Hira D, Osaki R, et al. Sorafenib exposure and its correlation with response and safety in advanced hepatocellular carcinoma: 
results from an observational retrospective study. Cancer Chemother. Pharmacol 2020;86:129-39.

58. Lee MS, RyooBY, Hsu CH, et al. Atezolizumab with or without bevacizumab in unresectable hepatocellular carcinoma (GO30140): an open-label, multicentre, phase 1b study. Lancet Oncol 2020;21:808-20.

59. Kim R, Tan E, Wang E, et al. A phase i trial of trametinib in combination with sorafenib in patients with advanced hepatocellular cancer. Oncologist 2020; doi: 10.1634/theoncologist.2020-0759.

60. Berenguer M, Burra P, Ghobrial M, et al. Posttransplant management of recipients undergoing liver transplantation for hepatocellular carcinoma. Working Group Report from the ILTS Transplant Oncology Consensus Conference. Transplantation 2020;1143-9.

61. Halazun KJ, Najjar M, Abdelmessih RM, et al. Recurrence after liver transplantation for hepatocellular carcinoma. Ann Surg 2017;265:557-64.

62. Lee JH, Cho Y, Kim HY, et al. Serum tumor markers provide refined prognostication in selecting liver transplantation candidate for hepatocellular carcinoma patients beyond the milan criteria. Ann Surg 2016;263:842-50.

63. Mazzaferro V, Sposito C, Zhou J, et al. Metroticket 2.0 model for analysis of competing risks of death after liver transplantation for hepatocellular carcinoma. Gastroenterology 2018;154:128-39. 